TU-463

April 5, 2018

\title{
Gravitino Decay into a Neutrino and a Sneutrino in the Inflationary Universe
}

\author{
M. Kawasaki \\ Institute for Cosmic Ray Research, The University of Tokyo, Tanashi 188, Japan \\ and \\ T. Moroi t \\ Department of Physics, Tohoku University, Sendai 980, Japan
}

\begin{abstract}
Gravitino produced in the inflationary universe are studied. When the gravitino decays into a neutrino and a sneutrino, the emitted high energy neutrinos scatter off the background neutrinos and produce charged leptons (mainly electrons and positrons), which cause the electro-magnetic cascades and produce many soft photons. We obtain the spectra of the high energy neutrinos as well as the spectrum of the high energy photon by integrating a set of Boltzmann equations. Requiring these photons should not alter the abundances of the light elements (D, $\left.{ }^{3} \mathrm{He},{ }^{4} \mathrm{He}\right)$ in the universe, we can set the stringent upperbound on the reheating temperature after the inflation. We find that $T_{R} \lesssim\left(10^{10}-10^{12}\right) \mathrm{GeV}$ for $m_{3 / 2} \sim(100 \mathrm{GeV}-1 \mathrm{TeV})$, which is more stringent than the constraints in the previous works.
\end{abstract}

\footnotetext{
${ }^{1}$ Fellow of the Japan Society for the Promotion of Science.
} 


\section{Introduction}

In models based on supergravity [1]], it has been pointed out that there exist some light particles whose interactions are suppressed by powers of the gravitational scale $M=$ $M_{P l} / \sqrt{8 \pi} \simeq 2.4 \times 10^{18} \mathrm{GeV}$. Such particles have nothing to do with collider experiments, but

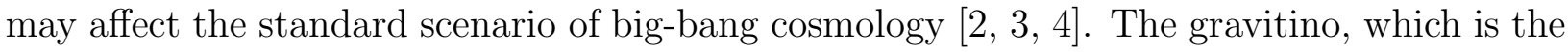
gauge field associated with local supersymmetry (SUSY), is one of the weakly interacting particles in supergravity models, and we expect that the mass of the gravitino is of order of typical SUSY-breaking scale.

Since the gravitino has only gravitational interaction with other particles, its lifetime is very long even if it is unstable. In standard cosmology, the gravitino with mass $m_{3 / 2} \sim$ $O(100 \mathrm{GeV}-10 \mathrm{TeV})$ is excluded since it decays after the big-bang nucleosynthesis (BBN) and produces an unacceptable amount of entropy, which conflicts with the predictions of BBN [2]. We may avoid this constraint by assuming the inflationary cosmology, in which the initial abundance of the gravitino is diluted by the exponential expansion of the universe [5]. However gravitinos are reproduced by scattering processes off the thermal radiation after the universe has been reheated. Since the number density of the secondary gravitino is proportional to the reheating temperature, upperbound on the reheating temperature should be imposed not to overproduce gravitinos [6] - [16].

In the recent paper [16], we consider the unstable gravitino which decays into photon $(\gamma)$ and photino $(\tilde{\gamma})$ and derive upperbound on the reheating temperature $T_{R}\left(T_{R} \lesssim 10^{6} \mathrm{GeV}\right.$ for $m_{3 / 2} \sim 100 \mathrm{GeV}, T_{R} \lesssim 10^{9} \mathrm{GeV}$ for $m_{3 / 2} \sim 1 \mathrm{TeV}$ ) by requiring the produced high energy photons should not alter the abundances of light elements synthesized in BBN. The above constraint seems to be very stringent since such low reheating temperature requires very small decay rate of the inflaton field. For example, in chaotic inflation with a inflaton whose interactions are suppressed by gravitational scale $M$, the inflaton decay rate is expected to be $\Gamma_{\text {inf }} \sim m_{\text {inf }}^{3} / M_{P l}^{2}$ with $m_{\text {inf }}$ being the inflaton mass, and hence the reheating temperature is estimated as 17

$$
T_{R} \sim 0.1 \sqrt{\Gamma_{i n f} M_{P l}} \sim 10^{9} \mathrm{GeV}
$$

requiring that the inflaton field should produce the density perturbations observed by COBE $\left(m_{\text {inf }} \sim 10^{13} \mathrm{GeV}\right)[18$.

However the constraints might become much weaker when the gravitino decays only into 
weakly interacting particles. In the particle content of the minimal SUSY standard model, the only candidates are neutrino and sneutrino.

In this letter, we assume that the gravitino decays only into a neutrino and a sneutrino and derive constraints on the reheating temperature. In this case, the emitted high energy neutrino may scatter off the background neutrino and produce an electron-positron (or muon-anti-muon) pair, which then produces many soft photons through electro-magnetic cascade processes and destruct the light elements. Since the interaction between high energy neutrinos and background neutrinos is weak, it seems that the destruction of the light elements is not efficient. In fact, Gratsias, Scherrer and Spergel [13] showed that the constraint is not so stringent for the case that the gravitino decays into a neutrino and a sneutrino. However the previous analysis seems to be incomplete in a couple of points. First Gratsias et.al. [13] totally neglect the secondary high energy neutrinos which are produced by neutrino-neutrino scattering. The effect of the secondary neutrino may be important for heavy gravitino $\left(m_{3 / 2} \gtrsim 1 \mathrm{TeV}\right)$. Second, they only study the case where the destruction of ${ }^{4} \mathrm{He}$ results in the overproduction of $\left({ }^{3} \mathrm{He}+\mathrm{D}\right)$. However, for the heavy gravitino which decays in early stage of the $\mathrm{BBN}$, the destruction of $\mathrm{D}$ is more important since the electromagnetic cascade process is so efficient that the energy of soft photons becomes less than the threshold of ${ }^{4} \mathrm{He}$. Furthermore, as pointed out in ref. [16], the previous estimation of the gravitino production in the reheating epoch is underestimated. Those effects which are not taken into account in ref. [13] may lead to more stringent constraint on the reheating temperature. Therefore, in this letter, we reexamine the decay of the gravitino into a neutrino and a sneutrino $\left(\psi_{\mu} \rightarrow \nu+\tilde{\nu}\right)$ with taking all relevant effect into account, and as a result, we have obtained more stringent upperbound on the reheating temperature.

\section{Neutrino-Neutrino Interaction}

The high energy neutrinos $(\nu)$ produced in the gravitino decay scatter off the thermal neutrino $\left(\nu_{b}\right)$ in the background by the following processes;

$$
\begin{aligned}
& \nu_{i}+\nu_{i, b} \rightarrow \nu_{i}+\nu_{i}, \\
& \nu_{i}+\bar{\nu}_{i, b} \rightarrow \nu_{i}+\bar{\nu}_{i}, \\
& \nu_{i}+\bar{\nu}_{i, b} \rightarrow \nu_{j}+\bar{\nu}_{j}, \\
& \nu_{i}+\nu_{j, b} \rightarrow \nu_{i}+\nu_{j},
\end{aligned}
$$




$$
\begin{aligned}
& \nu_{i}+\bar{\nu}_{j, b} \rightarrow \nu_{i}+\bar{\nu}_{j}, \\
& \nu_{i}+\bar{\nu}_{i, b} \rightarrow e^{-}+e^{+}, \\
& \nu_{i}+\bar{\nu}_{i, b} \rightarrow \mu^{-}+\mu^{+},
\end{aligned}
$$

where index $i$ and $j$ represent $e, \mu$ and $\tau$ with $i \neq j$. All the amplitude square $|\mathcal{M}|^{2}$ in these reactions take the form given by

$$
|\mathcal{M}|^{2}=32 G_{F}^{2}\left\{a\left(p p^{\prime}\right)^{2}+b(p q)^{2}+c\left(p q^{\prime}\right)^{2}+d m^{2}\left(p p^{\prime}\right)\right\},
$$

where $G_{F} \simeq 1.17 \times 10^{-5} \mathrm{GeV}^{-2}$ is the Fermi constant, the coefficients $a-d$ depend on the individual reaction, $p$ and $p^{\prime}$ are the initial momenta of high energy neutrino and background neutrino, $q$ and $q^{\prime}$ are the final momenta, and $m$ represents the mass of the fermion the in final state. Then one can obtain the Boltzmann equations describing the evolution of the spectra for the high energy neutrinos;

$$
\begin{aligned}
\frac{\partial f_{\nu_{i}}\left(E_{\nu}^{\prime}\right)}{\partial t}= & \frac{4 G_{F}^{2}}{3 \pi} \int_{E_{\nu}^{\prime}}^{\infty} \frac{d E_{\nu}}{E_{\nu}^{2}} \sum_{j}\left\{a_{i n, i j} E_{\nu}^{2}+b_{i n, i j}\left(E_{\nu}-E_{\nu}^{\prime}\right)^{2}+c_{i n, i j} E_{\nu}^{\prime 2}\right\} f_{\nu_{j}}\left(E_{\nu}\right) \\
& \times \int_{0}^{\infty} d \bar{E}_{\nu} \bar{E}_{\nu} \bar{f}_{\nu}\left(\bar{E}_{\nu}\right) \\
& -\frac{4 G_{F}^{2}}{3 \pi} E_{\nu}^{\prime} f_{\nu_{i}}\left(E_{\nu}^{\prime}\right)\left(a_{\text {out }}+\frac{1}{3} b_{\text {out }}+\frac{1}{3} c_{\text {out }}\right) \int_{0}^{\infty} d \bar{\epsilon}_{\nu} \bar{\epsilon}_{\nu} \bar{f}_{\nu}\left(\bar{\epsilon}_{\nu}\right) \\
& +\left(\frac{\partial f_{\nu_{i}}\left(E_{\nu}^{\prime}\right)}{\partial t}\right)_{\nu_{i}+\bar{\nu}_{i} \rightarrow e^{-}+e^{+}}+\left(\frac{\partial f_{\nu_{i}}\left(E_{\nu}^{\prime}\right)}{\partial t}\right)_{\nu_{i}+\bar{\nu}_{i} \rightarrow \mu^{-}+\mu^{+}} \\
& +\frac{1}{6 \tau_{3 / 2}} n_{3 / 2} \delta\left(E_{\nu}^{\prime}-m_{3 / 2} / 2\right) \\
& +E_{\nu}^{\prime} H \frac{\partial f_{\nu_{i}}\left(E_{\nu}^{\prime}\right)}{\partial E_{\nu}^{\prime}}-2 H f_{\nu_{i}}\left(E_{\nu}^{\prime}\right) E_{\nu}^{\prime},
\end{aligned}
$$

where $H$ is the expansion rate of the universe, coefficients $a-c$ are given by

$$
\begin{aligned}
& a_{\text {out }}=4, \quad b_{\text {out }}=0, \quad c_{\text {out }}=13, \\
& a_{\text {in,ii }}=6, \quad b_{\text {in }, i i}=9, \quad c_{i n, i i}=11, \\
& a_{\text {in }, i j}=1, \quad b_{\text {in, }, i j}=1, \quad c_{\text {in }, i j}=2, \quad(i \neq j),
\end{aligned}
$$

and the spectrum of the background neutrino $\bar{f}_{\nu}$ can be written as

$$
\bar{f}_{\nu}\left(\bar{E}_{\nu}\right)=\frac{\bar{E}_{\nu}^{2}}{2 \pi^{2}} \frac{1}{e^{\bar{E}_{\nu} / T_{\nu}}+1},
$$


with $T_{\nu}$ being the neutrino temperature. The last two terms of right-hand side of eq.(10) represent the effect of the cosmic expansion. The formula for charged lepton pair creation is obtained as

$$
\begin{aligned}
\left(\frac{f_{\nu_{i}}\left(E_{\nu}\right)}{\partial t}\right)_{\nu_{i}+\bar{\nu}_{i} \rightarrow l^{-}+l^{+}}= & -\frac{G_{F}^{2}}{16 \pi} \frac{1}{E_{\nu}^{2}} f_{\nu_{i}}\left(E_{\nu}\right) \int_{0}^{\infty} d \bar{E}_{\nu} \bar{f}_{\nu}\left(\bar{E}_{\nu}\right) \\
& \times\left\{\left(a+\frac{1}{3} b+\frac{1}{3} c\right) I_{2}+\left(2 d-\frac{1}{3} b-\frac{1}{3} c\right) m^{2} I_{1}\right\},
\end{aligned}
$$

with

$$
\begin{aligned}
I_{2}= & \frac{4}{3}\left\{4-\frac{4 m^{2}}{E_{\nu} \bar{E}_{\nu}}\right\}^{1 / 2} E_{\nu} \bar{E}_{\nu}\left(8 E_{\nu}^{2} \bar{E}_{\nu}^{2}-2 m^{2} E_{\nu} \bar{E}_{\nu}-3 m^{4}\right) \\
& -4 m^{6} \ln \left[\frac{2\left\{4-\left(4 m^{2} / E_{\nu} \bar{E}_{\nu}\right)\right\}^{1 / 2} E_{\nu} \bar{E}_{\nu}+4 E_{\nu} \bar{E}_{\nu}-2 m^{2}}{2 m^{2}}\right], \\
I_{1}= & 2\left\{4-\frac{4 m^{2}}{\left.E_{\nu} \bar{E}_{\nu}\right\}^{1 / 2} E_{\nu} \bar{E}_{\nu}\left(2 E_{\nu} \bar{E}_{\nu}-m^{2}\right)}\right. \\
& -2 m^{4} \ln \left[\frac{2\left\{4-\left(4 m^{2} / E_{\nu} \bar{E}_{\nu}\right)\right\}^{1 / 2} E_{\nu} \bar{E}_{\nu}+4 E_{\nu} \bar{E}_{\nu}-2 m^{2}}{2 m^{2}}\right] .
\end{aligned}
$$

Coefficients for the charged lepton production processes are given by

$$
\begin{aligned}
a_{\nu_{i}+\bar{\nu}_{i} \rightarrow l_{i}^{-}+l_{i}^{+}} & =0, \\
b_{\nu_{i}+\bar{\nu}_{i} \rightarrow l_{i}^{-}+l_{i}^{+}} & =16 \sin ^{4} \theta_{W}, \\
c_{\nu_{i}+\bar{\nu}_{i} \rightarrow l_{i}^{-}+l_{i}^{+}} & =16 \sin ^{4} \theta_{W}, \\
d_{\nu_{i}+\bar{\nu}_{i} \rightarrow l_{i}^{-}+l_{i}^{+}} & =16 \sin ^{4} \theta_{W}, \\
a_{\nu_{i}+\bar{\nu}_{i} \rightarrow l_{j}^{-}+l_{j}^{+}} & =0, \\
b_{\nu_{i}+\bar{\nu}_{i} \rightarrow l_{j}^{-}+l_{j}^{+}} & =16 \sin ^{4} \theta_{W}, \\
c_{\nu_{i}+\bar{\nu}_{i} \rightarrow l_{j}^{-}+l_{j}^{+}} & =\left(16 \sin ^{2} \theta_{W}-2\right)^{2}, \\
d_{\nu_{i}+\bar{\nu}_{i} \rightarrow l_{j}^{-}+l_{j}^{+}} & =16 \sin ^{4} \theta_{W}-8 \sin ^{2} \theta_{W},
\end{aligned}
$$

with $\theta_{W}$ being the Weinberg angle and $i \neq j$. The gravitino decay rate $\Gamma_{3 / 2}$ and the number density $n_{3 / 2}$ of the gravitino are given by

$$
\Gamma_{3 / 2}\left(\psi_{\mu} \rightarrow \nu+\tilde{\nu}\right)=\sum_{i} \frac{m_{3 / 2}^{3}}{192 \pi M}\left\{1-\left(\frac{m_{\tilde{\nu}_{i}}}{m_{3 / 2}}\right)^{2}\right\}^{4}
$$


and

$$
n_{3 / 2} \simeq 1.07 \times 10^{-11} n_{\gamma}\left(\frac{T_{R}}{10^{10} \mathrm{GeV}}\right)\left[1-0.0232 \log \left(\frac{T_{R}}{10^{10} \mathrm{GeV}}\right)\right] e^{-\Gamma_{3 / 2} t}
$$

where $m_{\tilde{\nu}_{i}}$ is the mass of the sneutrino of $i$-th generation and $n_{\gamma}$ is the photon number density. In the following analysis, we assume all the sneutrino masses are degenerate for simplicity.

In Fig. 17 the spectra of three types of neutrinos at $T=1 \mathrm{eV}$ are shown for $m_{3 / 2}=$ $100 \mathrm{GeV}, 1 \mathrm{TeV}$ and $10 \mathrm{TeV}$. As one can see, the differences among three types of neutrinos are very small since the differences only come from the charged lepton pair creation processes which are sub-dominant compared with the neutrino-neutrino scattering processes. For the heavy gravitino $\left(m_{3 / 2} \gtrsim 1 \mathrm{TeV}\right)$ the decay occurs early in the universe when the neutrino temperature is so high that the $\nu-\nu_{b}$ scatterings is effective. (Notice that the cross section is roughly given by $\langle\sigma v\rangle \sim G_{F}^{2} E_{\nu} T_{\nu}$.) Therefore high energy neutrinos emitted from the gravitinos scatter off the background neutrinos frequently and produce many secondary neutrinos. On the other hand, the emitted neutrinos seldom interact with the background neutrinos if the gravitino mass is small $\left(m_{3 / 2} \lesssim 100 \mathrm{GeV}\right)$ and the effect of the neutrinoneutrino scattering is negligible.

\section{High Energy Photon Spectrum}

The primary and secondary high energy neutrinos scatter off the background neutrinos and produce charged lepton pairs. Then the charged leptons induce the electro-magnetic cascade processes. In the same procedure as ref. [16] we obtain the high energy photon and electron spectra, taking the following radiative processes into account. (I) High energy photon with energy $\epsilon_{\gamma}$ can become electron-positron pair by scattering off background photon if the energy of the background photon is larger than $m_{e}^{2} / \epsilon_{\gamma}$ where $m_{e}$ is the electron mass. We call this process double photon pair creation. For sufficiently high energy photons, this is the dominant process since the cross section or the number density of target is much larger than other processes. Numerical calculation shows that this process determines the shape of the spectrum of high energy photon for $\epsilon_{\gamma} \gtrsim m_{e}^{2} / 22 T$. (II) Below the effective threshold of double photon pair creation, high energy photons lose their energy by photon-

\footnotetext{
${ }^{2}$ In the recent article, Fischler [19] have proposed a new mechanism to produce gravitinos in thermal bath. If one adopts his mechanism, the constraints obtained below must become more stringent. However, it is not clear to us if his new mechanism is relevant.
} 
photon scattering. But in the limit of $\epsilon_{\gamma} \rightarrow 0$, the total cross section for the photon-photon scattering is proportional to $\epsilon_{\gamma}^{3}$ and this process loses its significance. Hence finally, photons are thermalized by (III) pair creation in nuclei, or (IV) Compton scattering off background electron. And (V) emitted high energy electrons and positrons lose their energy by the inverse Compton scattering off background photon.

The Boltzmann equations for the photon and electron distribution function $f_{\gamma}$ and $f_{e}$ are given by

$$
\begin{aligned}
\frac{\partial f_{\gamma}\left(\epsilon_{\gamma}\right)}{\partial t}= & \left.\frac{\partial f_{\gamma}\left(\epsilon_{\gamma}\right)}{\partial t}\right|_{\mathrm{DP}}+\left.\frac{\partial f_{\gamma}\left(\epsilon_{\gamma}\right)}{\partial t}\right|_{\mathrm{PP}}+\left.\frac{\partial f_{\gamma}\left(\epsilon_{\gamma}\right)}{\partial t}\right|_{\mathrm{PC}} \\
& +\left.\frac{\partial f_{\gamma}\left(\epsilon_{\gamma}\right)}{\partial t}\right|_{\mathrm{CS}}+\left.\frac{\partial f_{\gamma}\left(\epsilon_{\gamma}\right)}{\partial t}\right|_{\mathrm{IC}}, \\
\frac{\partial f_{e}\left(E_{e}\right)}{\partial t}= & \left.\frac{\partial f_{e}\left(E_{e}\right)}{\partial t}\right|_{\mathrm{DP}}+\left.\frac{\partial f_{e}\left(E_{e}\right)}{\partial t}\right|_{\mathrm{PC}}+\left.\frac{\partial f_{e}\left(E_{e}\right)}{\partial t}\right|_{\mathrm{CS}} \\
& +\left.\frac{\partial f_{e}\left(E_{e}\right)}{\partial t}\right|_{\mathrm{IC}}+\left.\frac{\partial f_{e}\left(E_{e}\right)}{\partial t}\right|_{\mathrm{NEU}},
\end{aligned}
$$

where DP (PP, PC, CS, IC, and NEU) represents double photon pair creation (photonphoton scattering, pair creation in nuclei, Compton scattering, inverse Compton scattering, and the contribution from the $\nu-\nu$ scatterings). In solving eqs.(28) and (29), we assume

$$
\left.\frac{f_{e}(E)}{\partial t}\right|_{\mathrm{NEU}}=-\sum_{i}\left(\left.\frac{f_{\nu_{i}}(E)}{\partial t}\right|_{\nu_{i}+\bar{\nu}_{i} \rightarrow e^{-}+e^{+}}+\left.\frac{f_{\nu_{i}}(E)}{\partial t}\right|_{\nu_{i}+\bar{\nu}_{i} \rightarrow \mu^{-}+\mu^{+}}\right),
$$

which respects the energy conservation although the actual energy distribution may be different. This assumption is adequate for our purpose since the photon (and electron) spectrum is determined almost only by the total amount of the energy injection [16]. Furthermore we treat muons (and anti-muons) as electrons with the same energy and neglect the contribution from tau leptons whose creation rate is much smaller than the other charged leptons. Full details for other terms are shown in ref. 16.

\section{BBN and Photo-Dissociation of Light Elements}

The presence of gravitino might destroy the success of BBN. The gravitino may have three effects on BBN. First the energy density of the gravitino at $T \simeq 1 \mathrm{MeV}$ speeds up the cosmic expansion and leads to increase the $n / p$ ratio at the neutron decoupling time and hence ${ }^{4} \mathrm{He}$ abundance also increases. Second, the entropy production due to the decay of the 
gravitino reduces the baryon-to-photon ratio and results in too baryon-poor universe. Third, the high energy photons induced by the decay of the gravitino destroy the light elements $\left(\mathrm{D},{ }^{3} \mathrm{He},{ }^{4} \mathrm{He}\right.$ ). Among three effects, photo-dissociation of the light elements by high energy photon is the most important for the relatively light gravitino $\left(m_{3 / 2} \lesssim 1 \mathrm{TeV}\right)$. The entropy production is not important for the present case since gravitinos decay into invisible particles (i.e. neutrinos).

When the energy of the high energy photon is relatively low, i.e. $2 \mathrm{MeV} \lesssim \epsilon_{\gamma} \lesssim 20 \mathrm{MeV}$, the D, T and ${ }^{3} \mathrm{He}$ are destroyed and their abundances decrease. On the other hand, if the photons have high energy enough to destroy ${ }^{4} \mathrm{He}$, it seems that such high energy photons only decrease the abundance of all light elements. However since D, T and ${ }^{3} \mathrm{He}$ are produced by the photo-dissociation of ${ }^{4} \mathrm{He}$ whose abundance is much higher than the other elements, their abundances can increase or decrease depending on the number density of the high energy photon. When the number density of the high energy photons with energy greater than $\sim$ $20 \mathrm{MeV}$ is extremely high, all light elements are destroyed. But as the photon density becomes lower, there is some range of the high energy photon density at which the overproduction of $\mathrm{D}, \mathrm{T}$ and ${ }^{3} \mathrm{He}$ becomes significant. And if the density is sufficiently low, the high energy photon does not affect the BBN at all.

From various observations, the primordial abundances of light elements are estimated [20] as

$$
\begin{aligned}
& 0.22<Y_{p} \equiv\left(\frac{\rho^{4} \mathrm{He}}{\rho_{B}}\right)_{p}<0.24, \\
& \left(\frac{n_{\mathrm{D}}}{n_{\mathrm{H}}}\right)_{p}>1.8 \times 10^{-5}, \\
& \left(\frac{n_{\mathrm{D}}+n^{3} \mathrm{He}}{n_{\mathrm{H}}}\right)_{p}<1.0 \times 10^{-4},
\end{aligned}
$$

where $\rho^{4} \mathrm{He}$ and $\rho_{B}$ are the mass densities of ${ }^{4} \mathrm{He}$ and baryon. The abundances of light elements modified by the gravitino decay must satisfy the observational constraints above. In order to make precise predictions for the abundances of light elements, we have modified Kawano's computer code [21 to include the photo-dissociation processes.

In the present calculation there are at least three free parameters, i.e. the mass of the gravitino $m_{3 / 2}$, reheating temperature $T_{R}$ and the baryon-to-photon ratio $\eta_{B}$. However as shown in the previous paper [16], the baryon-to-photon ratio $\eta_{B}$ is not important parameter 
because the allowed value for $\eta_{B}$ is almost the same as that in the standard case (i.e. without gravitino). Therefore we fix $\eta_{B}=3 \times 10^{-10}$ in this letter.

\section{$5 \quad$ Results and Discussions}

The allowed regions that satisfy the observational constraints (31)-(33) is shown in the $m_{3 / 2}-T_{R}$ plane in Fig.2. In Fig.2 one can see that for the gravitino with mass between $\sim$ $100 \mathrm{GeV}$ and $\sim 1 \mathrm{TeV}$, the overproduction of $\mathrm{D}$ and ${ }^{3} \mathrm{He}$ gives the most stringent constraint, while the upperbound on the reheating temperature is determined from the destruction of $\mathrm{D}$ for $m_{3 / 2} \simeq(1-3) \mathrm{TeV}$. Notice that $\mathrm{D}$ destruction is not considered in the previous work [13]. Furthermore the constrain from $\left(\mathrm{D}+{ }^{3} \mathrm{He}\right)$ overproduction is more stringent. The reasons why we obtain the more stringent constrain are i) the gravitino abundance is (4-5) times higher than the one obtained in ref. 8 that the previous authors used, ii) the secondary neutrinos are taken into account in our calculation, and iii) the gravitino lifetime for $\psi_{\mu} \rightarrow \nu+\tilde{\nu}$ is longer by a factor 2 than the lifetime for $\psi_{\mu} \rightarrow \gamma+\tilde{\gamma}$. (In ref. 13] it is presumed that $\Gamma_{3 / 2}\left(\psi_{\mu} \rightarrow \nu+\tilde{\nu}\right)=\Gamma_{3 / 2}\left(\psi_{\mu} \rightarrow \gamma+\tilde{\gamma}\right)$.)

In Fig.2, the overproduction of ${ }^{4} \mathrm{He}$ due to the mass density of the gravitino at $T \sim 1 \mathrm{MeV}$ is also seen. The constraint from this effect is less stringent than those from the photodissociation of $\mathrm{D}$ and the present sneutrino mass density (discussed below).

If we assume that the sneutrino is the lightest SUSY particle, the sneutrinos produced by the gravitino decay are stable and their present mass density is given by

$$
\Omega_{\tilde{\nu}} h^{2}=0.037\left(\frac{m_{\tilde{\nu}}}{100 \mathrm{GeV}}\right)\left(\frac{T_{R}}{10^{10} \mathrm{GeV}}\right)
$$

where $h$ is the Hubble constant in units of $100 \mathrm{~km} / \mathrm{sec} / \mathrm{Mpc}$. Therefore the present limit on sneutrino mass $41 \mathrm{GeV}$ [22] sets the upperbound on the reheating temperature;

$$
T_{R} \leq 6.6 \times 10^{11} h^{2} \mathrm{GeV}
$$

This constraint is also shown in Fig.2. . $^{3}$

\footnotetext{
${ }^{3}$ In calculating eq.(34), we have ignored the effect of the pair annihilation of sneutrinos $\left(\tilde{\nu}+\tilde{\nu}^{*} \rightarrow f+\bar{f}\right)$. For the pair annihilation process, the cross section is roughly estimated as $\sigma v \lesssim \alpha_{W} / m_{\tilde{\nu}}^{2}$ (where $\alpha_{W}$ is the coupling factor and $v$ is the relative velocity), and the condition for sufficiently large pair annihilation rate $\left(n_{\tilde{\nu}} \sigma v \gtrsim H\right.$, with $n_{\tilde{\nu}}$ being the sneutrino density) reduces to $n_{\tilde{\nu}} / n_{\gamma} \gtrsim 10^{-7}$ (with $Y_{\tilde{\nu}}$ being the yield variable for the sneutrino), which is less stringent than the constraint (35). However, if the sneutrino mass is small enough to hit the pole of the $Z$-boson propagator, the constraint (35) becomes weaker, and constraint from the ${ }^{4} \mathrm{He}$ overproduction may become significant for large gravitino mass $\left(m_{3 / 2} \gtrsim 3 \mathrm{TeV}\right)$.
} 
In summary, we have investigated the decay of the gravitino into a neutrino and a sneutrino, in particular, the effect on BBN by high energy photons induced by the decay. We have found the constraint on reheating temperature and mass of the gravitino as

$$
T_{R} \lesssim 10^{10-12} \mathrm{GeV} \quad \text { for } \quad m_{3 / 2}=100 \mathrm{GeV}-10 \mathrm{TeV}
$$

which is more stringent than the one obtained in ref.[13], but compatible with the rough estimation of the reheating temperature (1) for a chaotic inflation.

\section{Acknowledgement}

We would like to thank T. Yanagida for useful discussions, and to K. Maruyama for informing us of the experimental data for photo-dissociation processes. One of the author (T.M.) thanks Institute for Cosmic Ray Research where part of this work has done. This work is supported in part by the Japan Society for the Promotion of Science. 


\section{References}

[1] E. Cremmer, S. Ferrara, L. Grardello and A. van Proeyen, Nucl. Phys. B212 (1983) 413.

[2] S. Weinberg, Phys. Rev. Lett. 48 (1982) 1303.

[3] L.M. Krauss, Nucl. Phys. B277 (1983) 556.

[4] G.D. Coughlan, N. Fischler, E.W. Kolb, S. Raby and G.G. Ross, Phys. Lett. B131 (1983) 59 .

[5] J. Ellis, A.D. Linde and D.V. Nanopoulos, Phys. Lett. B118(1982) 59.

[6] D.V. Nanopoulos, K.A. Olive and M. Srednicki, Phys. Lett. B127(1983) 30.

[7] M.Yu. Khlopov and A.D. Linde, Phys. Lett. B138(1984) 265.

[8] J. Ellis, E. Kim and D.V. Nanopoulos, Phys. Lett. B145 (1984) 181.

[9] R. Juszkiewicz, J. Silk and A. Stebbins, Phys. Lett. B158 (1985) 463.

[10] J. Ellis, D.V. Nanopoulos and S. Sarkar, Nucl. Phys. B259 (1985) 175.

[11] M. Kawasaki and K. Sato, Phys. Lett. B189 (1987) 23.

[12] V.S. Berezinsky, Phys. Lett. B261 (1991) 71.

[13] J. Gratsias, R.J. Scherrer and D.N. Spergel, Phys. Lett. B262 (1991) 198.

[14] J. Ellis, G.B. Gelmini, J.L. Lopez, D.V. Nanopoulos and S. Sarker, Nucl. Phys. B373 (1992) 399 .

[15] T. Moroi, H. Murayama and M. Yamaguchi, Phys. Lett. B303(1993) 289.

[16] M. Kawasaki and T. Moroi, preprint TU-457 (hep-ph9403364) (1994).

[17] E.W. Kolb and M.S. Terner, The Early Universe, (Addison-Wesley, 1990).

[18] D.S. Salopek, Phys. Rev. Lett. 69 (1992) 3602.

[19] W. Fischler, Phys. Lett. B332(1994) 277. 
[20] T.P. Walker, G. Steigman, D.N. Schramm, K.A. Olive and H.-S. Kang, Ap. J. 376 (1991) 51.

[21] L. Kawano, Fermilab preprint (1992).

[22] Particle Data Group, Phys. Rev. D45 (1992) S1. 


\section{Figure Captions}

Figure 1: Spectra of high energy electron-neutrino (solid curve), muon-neutrino (dashed curve) and tau-neutrino (dotted curve) at $T=1 \mathrm{eV}$ for $m_{3 / 2}=100 \mathrm{GeV}, 1 \mathrm{TeV}$ and $10 \mathrm{TeV}$. We have taken $T_{R}=10^{10} \mathrm{GeV}$.

Figure 2: Constraints on the reheating temperature from $\mathrm{BBN}$, the present mass density of the sneutrino. The region above the curves is excluded. The lower mass of the gravitino from $m_{3 / 2}>m_{\tilde{\nu}}>41 \mathrm{GeV}$ is also shown in long-short dashed line. 


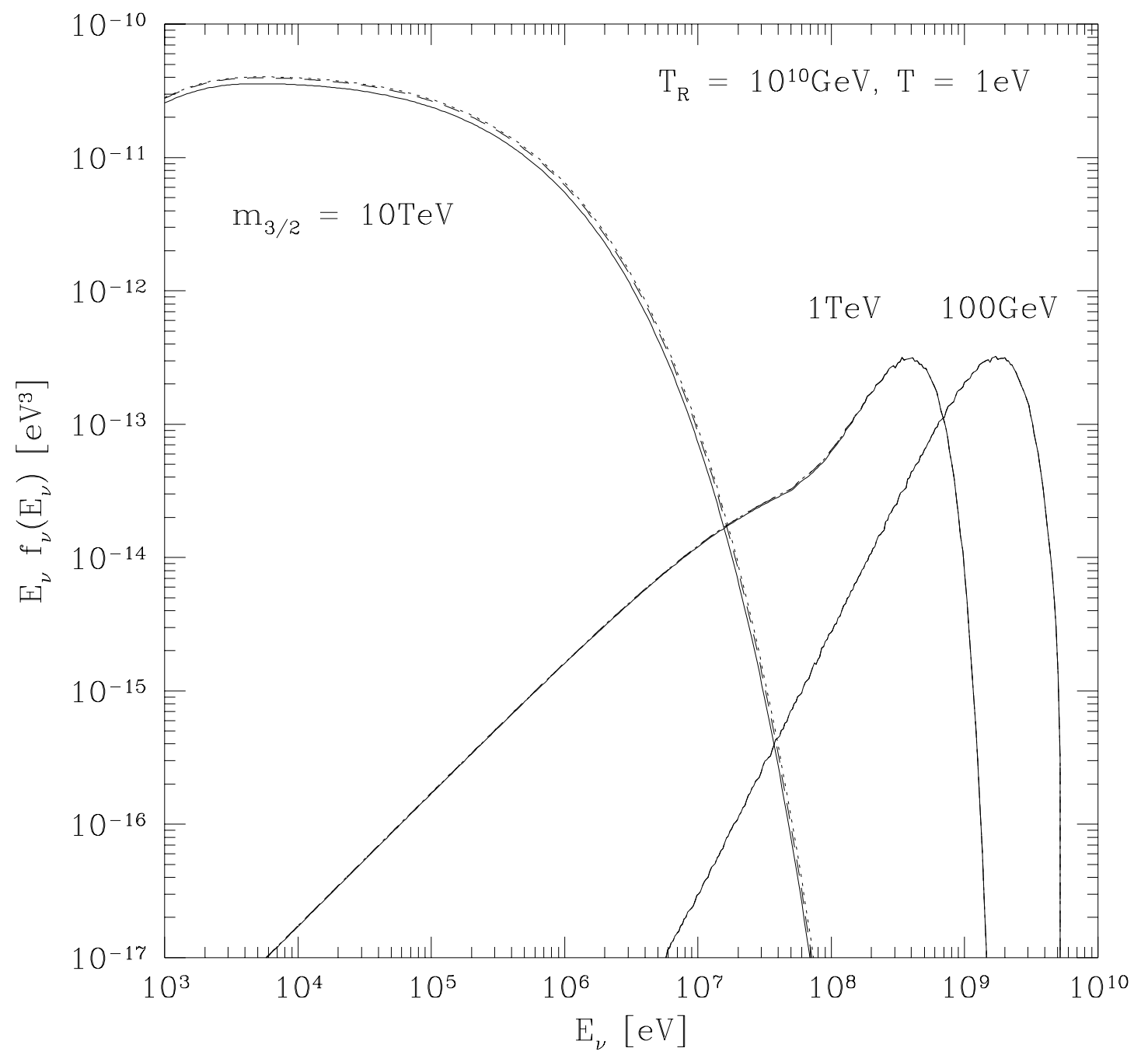

Fig. 1 


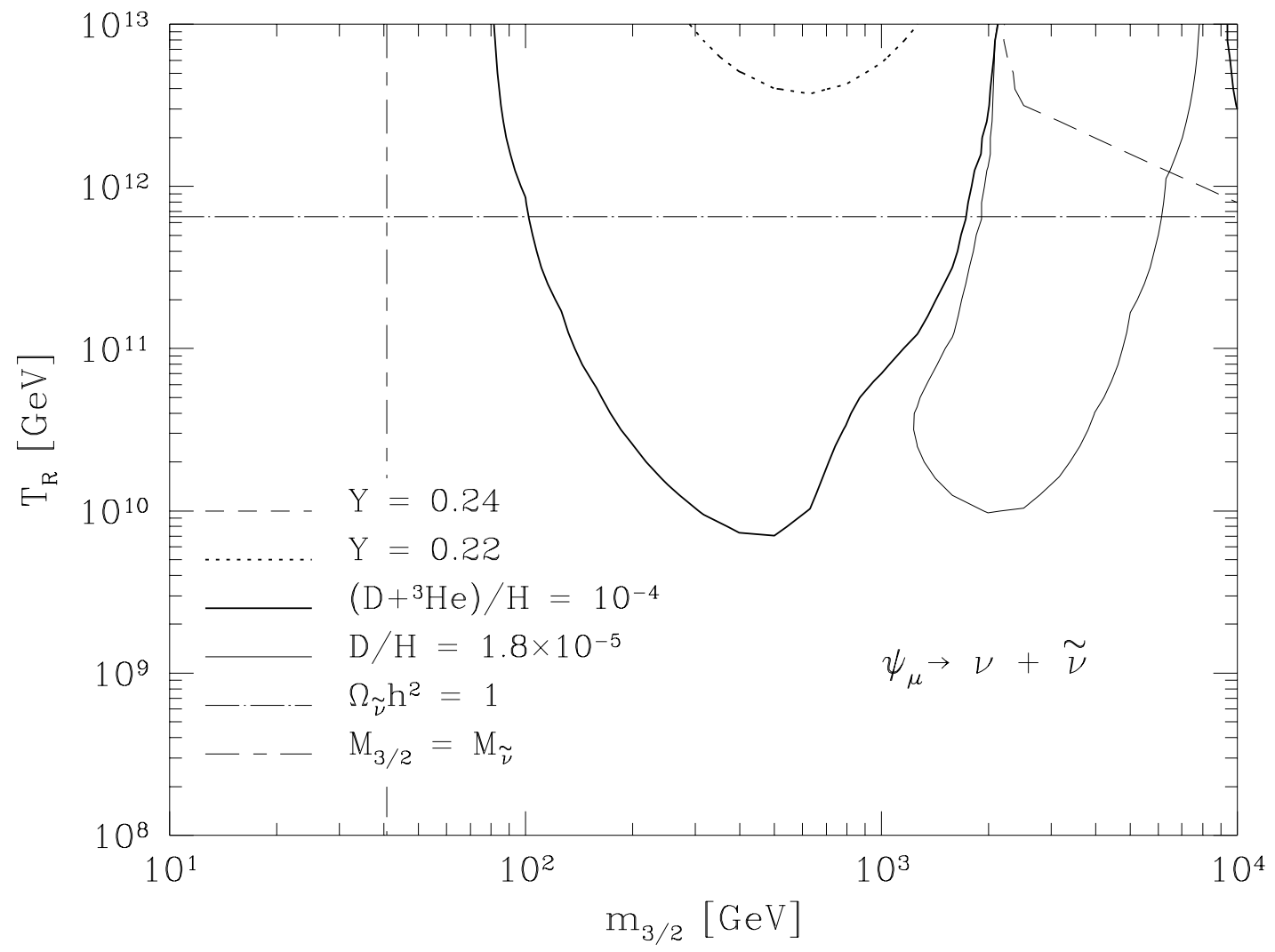

Fig. 2 